\title{
Enhancing the Specular Effect of Metallic Color Prints by Reducing the Use of Yellow Ink
}

\author{
Thomas Bugnon, Andrea Maesani, and Roger D. Hersch ${ }^{\wedge}$ \\ School of Computer and Communication Sciences, École Polytechnique \\ Fédérale de Lausanne (EPFL), Lausanne, Switzerland \\ E-mail:Thomas.bugnon@a3.epfl.ch
}

\begin{abstract}
The MetallicArt technique is used as a visual security feature to hide patterns in CMY images using an additional silver ink. The patterns are hidden under nonspecular viewing conditions and visible under specular viewing conditions. By varying the amount of silver ink, the authors control the intensity of the specular reflection of the printed pattern. Using a spectral reflection prediction model, they can constrain the maximization of the silver ink (S) in order to keep the color difference between the original CMY color and the fitted CMYS color under a given threshold, ensuring that the pattern is hidden under nonspecular viewing conditions. When the printing device requires the inks to be UV cured, the leafing effect of the silver ink is reduced. This also reduces the specular reflection of the silver ink, which becomes comparable to the specular reflection of the yellow ink. Since both specular reflections are similar, it is difficult to distinguish the embedded patterns under specular viewing conditions when both inks are present. The authors, therefore, propose a new algorithm to embed patterns in CMY images that not only maximizes silver but also minimizes yellow. Experimental results show that the proposed algorithm enhances the visibility of the patterns under specular viewing conditions, allowing the use of MetallicArt when the printing device requires UV curing. (C) 2011 Society for Imaging Science and Technology. [DOI: 10.2352/J.ImagingSci.Technol.2011.55.6.060506]
\end{abstract}

\section{INTRODUCTION}

When light hits a cyan, magenta, and yellow halftone print, it is partly absorbed by the ink layer and partly diffused by the paper substrate, except for the small specular reflection that occurs at the Fresnel interface between air and paper. Although this represents only an approximation of the real phenomena, it has been at the foundation of spectral reflection prediction models and works well in practice.

This assumption does not hold for metallic inks. These inks are opaque and reflect most of the light. The incident light does not reach the paper and remains within a relatively narrow cone. The resulting strong specular reflection has been used to hide metallic patterns in normal CMY images, a technique called MetallicArt. ${ }^{1}$ Using silver ink, this technique consists in determining the image pixels located within a given pattern and to replace their original CMY coverages by CMYS (cyan, magenta, yellow, and silver) coverages containing as much metallic ink as possible, while preserving the original CIELAB color under nonspecular viewing conditions. The

\section{IS\&T Member.}

Received Apr. 18, 2011; accepted for publication Sep. 30, 2011; published online Dec. 23, 2011

1062-3701/2011/55(6)/060506/9/\$20.00. preservation of the original color under nonspecular viewing conditions is achieved using a spectral reflection prediction model. The pattern is thus invisible under nonspecular viewing conditions but becomes visible under specular viewing conditions, thanks to the presence of the silver ink.

The MetallicArt technique works well when the leafing effect is strong, i.e., when the metallic particles present in the metallic ink align themselves horizontally along the surface of the ink film. ${ }^{2}$ This effect is, however, reduced when printing with a web offset press. Due to the speed of such presses and to avoid smearing the press, the metallic ink must be dried as soon as printed, a process called UV curing. This fixates the metallic particles on the paper substrate before the leafing effect can fully take place, reducing the specular reflection of the metallic ink. Figure 1 shows the impact of UV curing. In Fig. 1(a), the leafing effect is reduced due to UV curing and the metallic particles cover much less of the surface than in Fig. 1(b) where the ink is dried without UV curing.

This reduced specular reflection becomes a problem in regions largely covered by yellow. The yellow ink is not entirely transparent and slightly scatters light, showing a surprisingly strong specular reflection. This specular reflection competes with the specular reflection of the metallic ink and reduces the visibility of the embedded pattern under specular viewing conditions.

In this contribution, we first propose a simplified CMYS spectral prediction model for the MetallicArt technique and compare its prediction accuracy with the accuracy of the more complex CMYS model described in Ref. 1. We, then, show that the specular reflection of the silver ink is not visible in the presence of yellow. We, therefore, propose an algorithm that minimizes the coverage of yellow in order to increase the visibility of the embedded metallic patterns under specular viewing conditions.

\section{EXPERIMENTAL DETAILS}

All the experiments are performed on a Müller Martini Concept web offset press using the cyan (43UE5200), magenta (42UE5200), and yellow (41UE5200) standard process inks from Stehlin+Hostag (NewV set MFX) and metallic silver ink SICPA 367701. The paper used is a mat coated Artic silk of $170 \mathrm{gr} / \mathrm{m} 2$. We use classical rotated halftone screens at 150 lpi imaged at $2400 \mathrm{dpi}$ with angles of $75^{\circ}, 15^{\circ}, 0^{\circ}$, and $45^{\circ}$ for the cyan, magenta, yellow, and silver inks, respectively. 


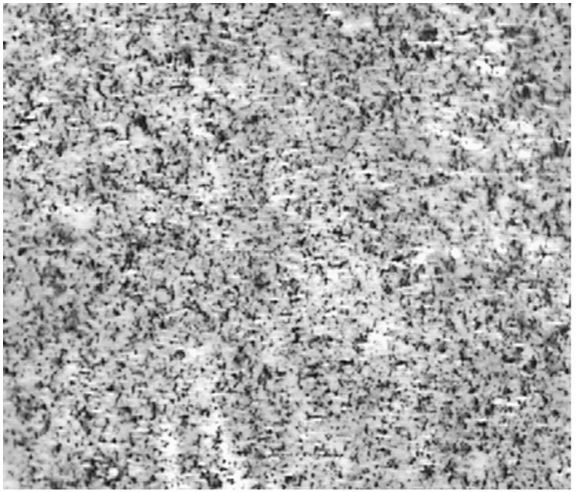

(a)

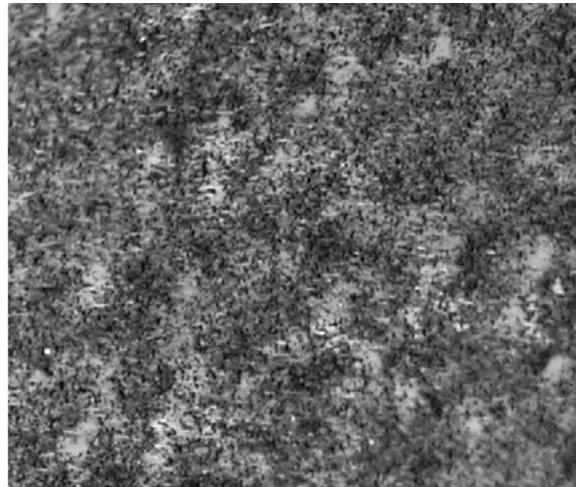

(b)

Figure 1. Microscope images of layers of solid silver ink when printed on: (a) a web offset press requiring UV curing and (b) a Komori sheetfed offset press that does not use UV curing. On average, the metallic ink covers $33 \%$ of the surface with UV curing and $60 \%$ without UV curing.

We performed measurements of the specular reflectance factors using a $\left(0^{\circ}: 0^{\circ}\right)$ geometry using the following Ocean Optics products. The light source is DH-2000-BAL's halogen lamp. We used a $230 \mu \mathrm{m}$ fiber optic reflection probe (QR2307-XSR) with a RPH-1 probe holder to illuminate the sample and collect the reflected light. The reflected light was measured with a Maya 2000 Pro spectrophotometer. Spectralon was used as the diffuse reference white. All reflectance factors were calculated in respect to this reference white.

\section{INK SPREADING ENHANCED YULE-NIELSEN MODIFIED SPECTRAL NEUGEBAUER MODEL (IS-YNSN)}

In order to preserve the original color of a CMY patch under nonspecular viewing conditions, while adding as much silver ink as possible, we use a spectral reflection prediction model. This model must be able to accurately predict the nonspecular reflectance of any CMYS patch. The original MetallicArt technique is based on an extension of the Clapper-Yule spectral reflection prediction model. ${ }^{3}$ This extension incorporates special parameters to take into account specificities of the silver ink such as large ink trapping and a raised metallic reflection spectrum in the presence of other inks. ${ }^{1}$ These specificities increase the complexity of the model. We further refer to this model as the Clapper-Yule based MetallicArt model (CY-MArt). We propose instead to use the simpler Yule-Nielsen model enhanced with our ink spreading model. ${ }^{4}$ We first present the Yule-Nielsen modified Spectral Neugebauer model (YNSN) before describing the ink spreading model. The prediction accuracy of the resulting IS-YNSN is then compared to the prediction accuracy of the original CY-MArt model.

\section{The Yule-Nielsen Modified Spectral Neugebauer Model (YNSN)}

One of the first color prediction models is the Neugebauer model. ${ }^{5}$ In its original form, it predicts the RGB values of a color halftone patch. Yule and Nielsen modified this model to account for optical dot gain, ${ }^{6}$ Yule and Colt applied it for CIE-XYZ tristimulus values, ${ }^{7}$ and Viggiano extended it to the spectral domain. ${ }^{8}$ This model, now known as the Yule-Nielsen modified Spectral Neugebauer model (YNSN), predicts the reflection spectra of color-constant patches, whose ink coverages are given. The equation used to perform a prediction is the following:

$$
R_{\text {pred }}(\lambda)=\left(\sum_{i} a_{i} R_{i}(\lambda)^{1 / n}\right)^{n},
$$

where $R_{\text {pred }}(\lambda)$ is the predicted reflection spectrum, $R_{i}(\lambda)$ is the reflection spectra of the colorants (also called Neugebauer primaries), $a_{i}$ is the relative area coverages of the colorants, and $n$ is the Yule-Nielsen $n$-value.

The spectra of the colorants must be measured and the scalar $n$-value needs to be calibrated. The relative area coverages of the colorants are computed from the relative ink surface coverages using the Demichel equations. ${ }^{9}$ For four inks, the Demichel equations are the following:

$$
\begin{aligned}
& a_{w}=(1-\mathrm{c}) \cdot(1-\mathrm{m}) \cdot(1-\mathrm{y}) \cdot(1-\mathrm{s}) \\
& a_{c}=c \cdot(1-\mathrm{m}) \cdot(1-\mathrm{y}) \cdot(1-\mathrm{s}) \\
& a_{m}=(1-c) \cdot m \cdot(1-y) \cdot(1-s) \\
& a_{c m}=c \cdot m \cdot(1-\mathrm{y}) \cdot(1-\mathrm{s}) \\
& a_{y}=(1-\mathrm{c}) \cdot(1-\mathrm{m}) \cdot y \quad \cdot(1-\mathrm{s}) \\
& a_{c y}=c \cdot(1-\mathrm{m}) \cdot y \cdot(1-\mathrm{s}) \\
& a_{m y}=(1-c) \cdot m \cdot y \cdot(1-s) \\
& a_{c m y}=c \cdot m \cdot y \cdot(1-s) \\
& a_{s}=(1-\mathrm{c}) \cdot(1-\mathrm{m}) \cdot(1-\mathrm{y}) \cdot s \\
& a_{c s}=c \cdot(1-\mathrm{m}) \cdot(1-\mathrm{y}) \cdot s \\
& a_{m s}=(1-\mathrm{c}) \cdot \mathrm{m} \cdot(1-\mathrm{y}) \cdot s \\
& a_{c m s}=c \cdot m \cdot(1-\mathrm{y}) \cdot s \\
& a_{y s}=(1-\mathrm{c}) \cdot(1-\mathrm{m}) \cdot y \cdot s^{\prime} \\
& a_{c y s}=c \cdot(1-\mathrm{m}) \cdot y \cdot s \\
& a_{m y s}=(1-\mathrm{c}) \cdot m \cdot y \cdot s \\
& a_{c m y s}=c \cdot m \cdot y \cdot s
\end{aligned}
$$


where $c, m, y$, and $s$ are the ink surface coverages of the cyan, magenta, yellow, and silver inks, respectively.

When calibrated, the YNSN model can be seen as a function taking the ink surface coverages of a halftone as input and yielding its reflection spectrum. It is possible to deduce ink coverages from a given reflection spectrum by minimizing a difference metric between the predicted and measured reflection spectra as follows:

$$
\operatorname{covs}_{\text {opt }}=\underset{\text { covs }}{\arg \min }\left[\sum_{\lambda}\left(R_{\text {pred }}(\lambda, \text { covs })-R(\lambda)\right)^{2}\right],
$$

where covs $=\{c, m, y, s\}$ are the ink coverages, $R(\lambda)$ is the measured spectrum, and $R_{\text {pred }}(\lambda)$ is the spectrum predicted by the YNSN model combining Eqs. (1) and (2).

Due to physical dot gain, ink coverages deduced using Eq. (3) are usually larger than the corresponding nominal ink coverages (see "The Ink Spreading Model” section). As a consequence, spectral predictions made with nominal ink coverages are not accurate. In order to make accurate predictions, the YNSN model should be augmented with an ink spreading model.

\section{The Ink Spreading Model}

When a reproduction device reproduces a color with given nominal ink coverages by depositing inks on paper, the inks spread out on the paper, resulting in effective coverages usually larger than the requested nominal ink coverages. This phenomenon is known as physical dot gain or ink spreading. The amount of dot gain of an ink depends on whether the ink halftone is printed alone on paper or in superposition with one or more other inks. The goal of the ink spreading model is therefore to deduce effective ink coverages from nominal ink coverages. The effective surface coverages are then used as input to the YNSN model.

\section{Ink Spreading Curves}

The ink spreading model relies on ink spreading curves (Figure 2(a)). An ink spreading curve maps the nominal surface coverages of an ink halftone into its effective surface coverages, i.e., to the surface that the ink halftone effectively covers once printed. We can approximate an ink spreading curve by a parabola characterized by its midpoint $v$ as follows:

$$
\begin{aligned}
f_{i / j k}\left(u_{i}, v_{i / j k}\right) & =u_{i}+\left(4 v_{i / j k}-2\right)\left(1-u_{i}\right) u_{i} \\
& =u_{i}^{2}\left(2-4 v_{i / j k}\right)+u_{i}\left(4 v_{i / j k}-1\right),
\end{aligned}
$$

where $u_{i}$ is the nominal surface coverage of ink halftone $i$, $f_{i / j k}$ is the ink spreading curve of ink halftone $i$ superposed with solid inks $j$ and $k$, and $v_{i / j k}$ is the effective surface coverage at $50 \%$ nominal surface coverage, also called midpoint of the ink spreading curve. Equation (4) represents a parabola passing through the three points $(0,0),\left(0.5, v_{i / j k}\right)$,

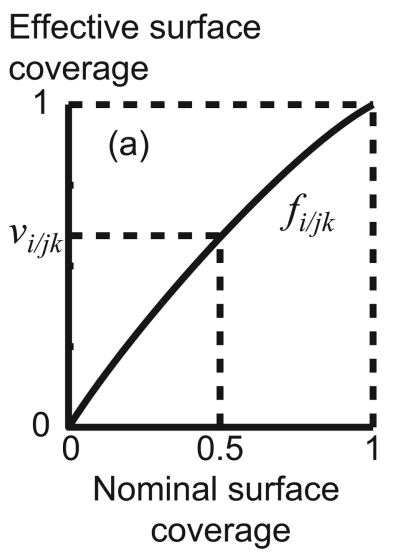

Dot gain

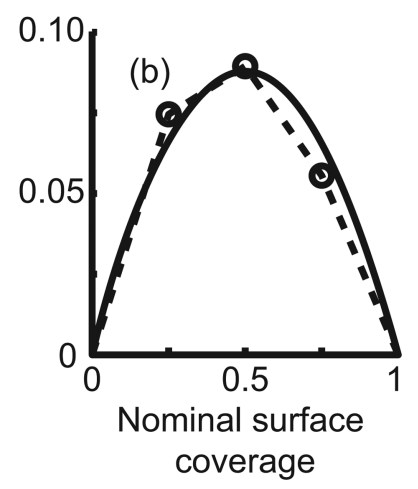

Figure 2. (a) Example of an ink spreading curve characterized by its midpoint $v$. (b) Corresponding dot gain curve defined as the difference between effective and nominal surface coverages, with the fitted dot gains of three calibration patches (circles), the linearly interpolated dot gain curve (dashed line), and the corresponding least-squares approximated parabolic dot gain curve (solid line).

Table I. List of ink spreading curve indicia.

\begin{tabular}{lccccccc}
\hline \multicolumn{2}{c}{ Cyan } & \multicolumn{2}{c}{ Magenta } & \multicolumn{2}{c}{ Yellow } & \multicolumn{2}{c}{ Silver } \\
\hline$c$ & $c / s$ & $m$ & $m / s$ & $y$ & $y / s$ & $s$ & $s / y$ \\
$c / m$ & $c / s m$ & $m / c$ & $m / s c$ & $y / c$ & $y / s c$ & $s / c$ & $s / c y$ \\
$c / y$ & $c / s y$ & $m / y$ & $m / s y$ & $y / m$ & $y / s m$ & $s / m$ & $s / m y$ \\
$c / m y$ & $c / s m y$ & $m / c y$ & $m / s c y$ & $y / c m$ & $y / s c m$ & $s / c m$ & $s / c m y$ \\
\hline
\end{tabular}

and $(1,1)$, as shown in Fig. 2(a). There is one ink spreading curve for each ink halftone in each superposition condition. For example, a cyan halftone may be printed alone, $c$, superposed with solid magenta, $c / m$; with solid yellow, $c / y$; with solid silver, $c / s$; with solid magenta and solid yellow, $c / m y$; with solid magenta and solid silver, $c / m s$; with solid yellow and solid silver, $c / y s$; and with solid magenta, yellow, and silver, $c / m y s$. There are eight different ink spreading curves for each ink, yielding a total of 32 ink spreading curves listed in Table I.

Fig. 2(b) shows the dot gain curve corresponding to the ink spreading curve of Fig. 2(a), where dot gain is defined as the difference between effective and nominal coverages. The ink spreading curve is calibrated using three calibration patches at $0.25,0.5$, and 0.75 nominal surface coverages. The nominal coverage of the ink halftone determines the position on the $\mathrm{x}$-axis of the ink spreading curve. The corresponding effective surface coverage is fitted using Eq. (3) and determines the position on the y-axis. The parabola is then fitted by minimizing the least-squares distance between the fitted effective surface coverages and that parabola. An ink spreading curve can be entirely characterized by the halftone at $50 \%$ nominal surface coverage, but as shown is Fig. 2(b), more calibration patches per ink spreading curve can be used. In the following experiments, 


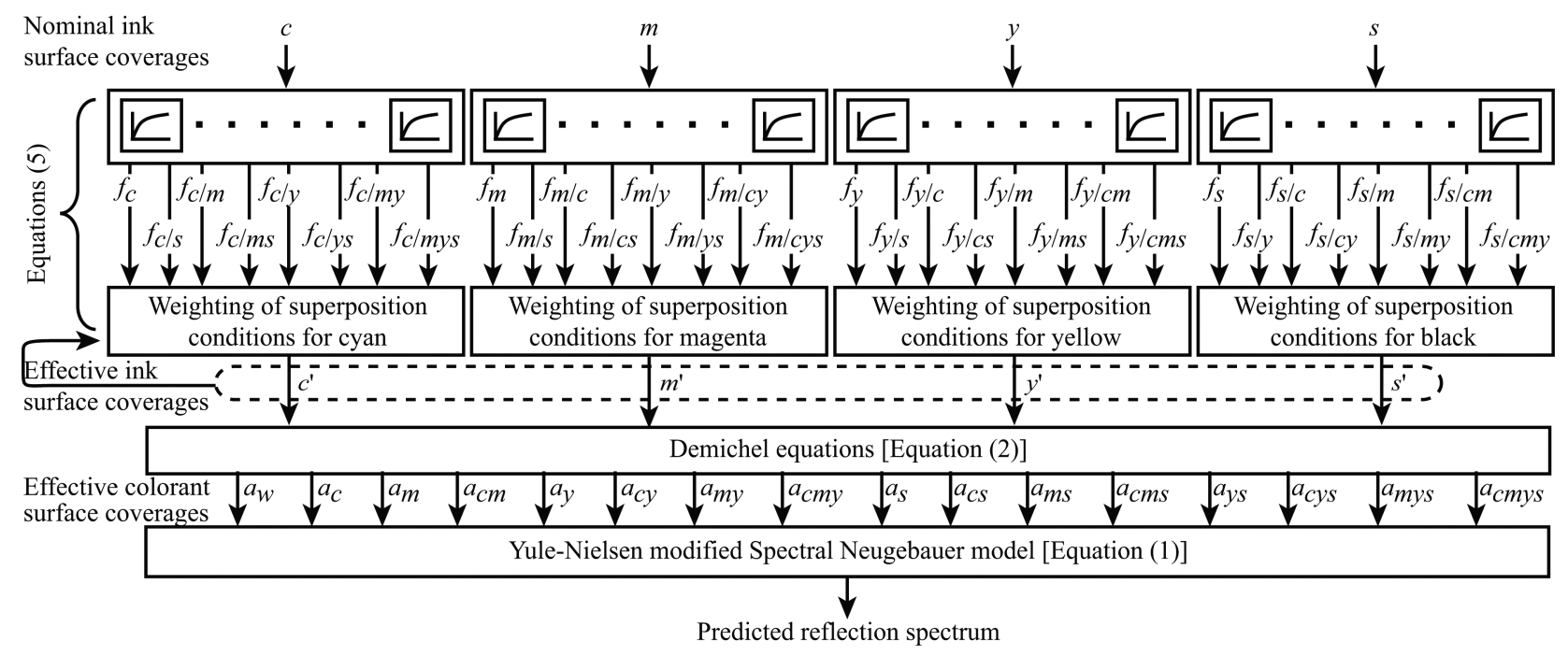

Figure 3. The ink spreading enhanced Yule-Nielsen modified Spectral Neugebauer model with nominal ink surface coverages $c, m, y$, and $s$; ink spreading curves $f_{i / j k}$ of ink $i$ superposed with solid inks $j$ and $k$; effective ink surface coverages $c^{\prime}, m^{\prime}, y$, and $s^{\prime}$; and effective colorant surface coverages $a_{w}$ to $a_{c m y s}$.

four nominal halftone surface coverages are used: $20 \%$, $40 \%, 60 \%$, and $80 \%$.

\section{Ink Spreading Equations}

In order to obtain the effective surface coverages of the ink dots forming a color halftone, we weight the contributions of the different ink spreading curves as follows. The effective coverage of ink halftone $i$ superposed with ink halftones $j$ and $k$ is the weighted average of the ink spreading functions $f_{i}, f_{i / j}, f_{i / k}$ and $f_{i / j k}$. The weights are computed according to the surface coverages of the underlying colorants (Neugebauer primaries) formed by inks $j$ and $k$. For CMYS, the ink spreading equations are

$$
\begin{array}{rlccll}
c^{\prime} & = & \left(1-m^{\prime}\right) & \left(1-y^{\prime}\right) & \left(1-s^{\prime}\right) & f_{c}(c) \\
& + & m^{\prime} & \left(1-y^{\prime}\right) & \left(1-s^{\prime}\right) & f_{c / m}(c) \\
& + & \left(1-m^{\prime}\right) & y^{\prime} & \left(1-s^{\prime}\right) & f_{c / y}(c) \\
& + & m^{\prime} & y^{\prime} & \left(1-s^{\prime}\right) & f_{c / m y}(c) \\
& + & \left(1-m^{\prime}\right) & \left(1-y^{\prime}\right) & s^{\prime} & f_{c / s}(c) \\
& + & m^{\prime} & \left(1-y^{\prime}\right) & s^{\prime} & f_{c / m s}(c) \\
& + & \left(1-m^{\prime}\right) & y^{\prime} & s^{\prime} & f_{c / y s}(c) \\
& + & m^{\prime} & y^{\prime} & s^{\prime} & f_{c / m y s}(c) \\
y^{\prime} & =\left(1-c^{\prime}\right) & \left(1-m^{\prime}\right) & \left(1-s^{\prime}\right) & f_{y}(y) \\
& + & c^{\prime} & \left(1-m^{\prime}\right) & \left(1-s^{\prime}\right) & f_{y / c}(y) \\
& + & \left(1-c^{\prime}\right) & m^{\prime} & \left(1-s^{\prime}\right) & f_{y / m}(y) \\
& + & c^{\prime} & m^{\prime} & \left(1-s^{\prime}\right) & f_{y / c m}(y) \\
& + & \left(1-c^{\prime}\right) & \left(1-m^{\prime}\right) & s^{\prime} & f_{y / s}(y) \\
+ & c^{\prime} & \left(1-m^{\prime}\right) & s^{\prime} & f_{y / c s}(y) \\
+ & \left(1-c^{\prime}\right) & m^{\prime} & s^{\prime} & f_{y / m s}(y) \\
+ & c^{\prime} & m^{\prime} & s^{\prime} & f_{y / c m s}(y)
\end{array}
$$

$$
\begin{aligned}
& m^{\prime}=\left(\begin{array}{llll}
\left(1-c^{\prime}\right) & \left(1-y^{\prime}\right) & \left(1-s^{\prime}\right) & f_{m}(m)
\end{array}\right. \\
& +\begin{array}{cccc}
c^{\prime} & \left(1-y^{\prime}\right) & \left(1-s^{\prime}\right) & f_{m / c}(m)
\end{array} \\
& +\left(1-c^{\prime}\right) \quad y^{\prime} \quad\left(1-s^{\prime}\right) \quad f_{m / y}(m) \\
& +\quad c^{\prime} \quad y^{\prime} \quad\left(1-s^{\prime}\right) \quad f_{m / c y}(m) \\
& +\left(1-c^{\prime}\right) \quad\left(1-y^{\prime}\right) \quad s^{\prime} \quad f_{m / s}(m) \\
& +\begin{array}{cccc}
c^{\prime} & \left(1-y^{\prime}\right) \quad s^{\prime} \quad f_{m / c s}(m)
\end{array} \\
& +\left(1-c^{\prime}\right) \quad y^{\prime} \quad s^{\prime} \quad f_{m / y s}(m) \\
& +\begin{array}{cccc}
c^{\prime} & y^{\prime} & s^{\prime} & f_{m / c y s}(m)
\end{array} \\
& s^{\prime}=\left(\begin{array}{llll}
\left(1-c^{\prime}\right) & \left(1-m^{\prime}\right) & \left(1-y^{\prime}\right) & f_{s}(s)
\end{array}\right. \\
& +\quad \begin{array}{cccc}
c^{\prime} & \left(1-m^{\prime}\right) & \left(1-y^{\prime}\right) & f_{s / c}(s)
\end{array} \\
& +\left(1-c^{\prime}\right) \quad m^{\prime} \quad\left(1-y^{\prime}\right) \quad f_{s / m}(s) \\
& +\quad c^{\prime} \quad m^{\prime} \quad\left(1-y^{\prime}\right) \quad f_{s / c m}(s) \\
& +\left(1-c^{\prime}\right)\left(1-m^{\prime}\right) \quad y^{\prime} \quad f_{s / y}(s) \\
& +c^{\prime} \quad\left(1-m^{\prime}\right) \quad y^{\prime} \quad f_{s / c y}(s) \\
& +\left(1-c^{\prime}\right) \quad m^{\prime} \quad y^{\prime} \quad f_{s / m y}(s) \\
& +c^{\prime} \quad m^{\prime} \quad y^{\prime} \quad f_{s / c m y}(s)
\end{aligned}
$$

We solve Eqs. (5) iteratively, starting by assigning the nominal ink halftone coverages $(c, m, y, s)$ to the effective ink halftone coverages $\left(c^{\prime}, m^{\prime}, y^{\prime}, s^{\prime}\right)$. Four to five iterations ensure sufficient convergence to determine the effective ink halftone surface coverages, ${ }^{4}$ i.e., the norm of the difference between two successive iterations is less than 0.001 .

\section{Comparison of the IS-YNSN and Prior Art CY-MArt Models}

Spectral predictions using the IS-YNSN model are performed according to Figure 3. For given nominal ink surface coverages $c, m, y$, we obtain effective surface coverages $c^{\prime}, m^{\prime}, y^{\prime}$, $s^{\prime}$ by weighting the ink spreading curves $f_{c}(c)$ to $f_{s / c m y}(s)$ 
Table II. Prediction accuracy of the CY-MArt and IS-YNSN models when predicting the 625 CMYS set.

\begin{tabular}{lccc} 
& \multicolumn{3}{c}{$\Delta \mathrm{E}_{94}$} \\
\cline { 2 - 4 } Model & Mean & $95 \%$ & Max \\
\hline CY-MArt & 2.49 & 6.24 & 15.55 \\
IS-YNSN & 1.69 & 3.55 & 6.19 \\
\hline
\end{tabular}

according to the surface coverages of the colorants contributing to that color halftone [Eqs. (5)]. With the Demichel equations, we then compute the corresponding effective surface coverages of the colorants forming that color halftone. With these effective colorant coverages, the YNSN model calculates the predicted reflection spectrum.

Before comparing the accuracy of the IS-YNSN model and the prior art CY-MArt model, ${ }^{1}$ we must calibrate these models. The calibration patches of the CY-MArt and ISYNSN models are composed of the 16 solid colorants (Neugebauer primaries) as well as 4 patches for each ink spreading curve, i.e., the ink spreading curves are calibrated at $20 \%, 40 \%, 60 \%$, and $80 \%$. The Yule-Nielsen $n$-value is set to 3.5 and the CY-MArt $b$-value to 0.45 . We then compare the accuracy of the IS-YNSN and CY-MArt models using a 625 CMYS patches test set composed of all possible combinations of cyan, magenta, yellow, and silver at $0 \%$, $25 \%, 50 \%, 75 \%$, and $100 \%$. Both the calibration and test patches are printed using the experimental setup described in the "Introduction" section and measured using the X-Rite SpectroEye spectrophotometer with a $\left(45^{\circ}: 0^{\circ}\right)$ geometry, illuminant D65 and $2^{\circ}$ observer.

Table II shows the results of predicting the 625 CMYS test set with the CY-MArt and IS-YNSN models. The present experiment shows that the IS-YNSN model is more accurate than the prior art CY-MArt model. Although much simpler, the IS-YNSN model better accounts for trapping or raised metallic spectrum thanks to its more advanced ink spreading model and unified approach to spectral prediction, i.e., the same prediction model is used for predicting reflectance factors for CMY alone and for CMY on top of solid silver.

\section{COMPARISON OF THE SPECULAR REFLECTANCE FACTORS OF THE YELLOW AND SILVER INKS}

The results obtained when printing images with embedded metallic patterns were not satisfactory on the web offset press described above. The metallic patterns were indeed invisible under nonspecular viewing conditions but were also hardly noticeable under specular viewing conditions. The fact that the patterns were hidden proves that the IS-YNSN model performs accurate predictions of the CMYS patches. We, therefore, investigated the specular viewing conditions and discovered that the most problematic regions were all largely covered with yellow ink.

For a pattern to appear under specular viewing conditions, there must be a noticeable difference between the original CMY color and the color containing additional silver. Figure 4 shows the reflectance factors of (a) solid cyan, (b) solid magenta, and (c) solid yellow printed on paper or on top of the solid silver ink. For cyan and magenta, the additional layer of solid silver ink noticeably increases the specular reflection. This is not the case for yellow. Yellow has a specular reflection above $500 \mathrm{~nm}$ as strong as the specular reflection of silver. Since there is no noticeable difference between the regions with or without silver, the hidden pattern is not revealed under specular viewing conditions.

Figure 5 shows the impact of reducing the coverage of yellow. Fig. 5(c) shows the specular reflectance factors of $80 \%$ of yellow when printed either on paper or on top of solid silver. In this case, the difference between the two specular reflections begins to be noticeable, especially below $500 \mathrm{~nm}$. The more we reduce the amount of yellow, the more noticeable the difference becomes.

These measured specular reflectance factors show the importance of reducing the amount of yellow as much as possible when adding the silver ink. In specular viewing conditions, this reduction increases the difference between regions with and without silver. However, it must be performed in such a way that these regions remain undistinguishable under nonspecular viewing conditions.

\section{REDUCING YELLOW BY CONVERTING CMY COVERAGES TO CMYS}

When embedding metallic patterns in an image, the pattern must be hidden under nonspecular viewing conditions and should reveal itself only under specular viewing condition. For the pattern to reveal itself under specular viewing conditions, it must contain as much silver ink and as little yellow ink as possible.

The conversion from the original CMY color to CMYS is performed using the CMYS version of the IS-YNSN model. This model is calibrated under a nonspecular $\left(45^{\circ}: 0^{\circ}\right)$ geometry using illuminant D65 and the $2^{\circ}$ standard observer for the current combination of inks, paper, and printing technology. When the model is calibrated, it can predict the nonspecular reflectance factors of both CMY and CMYS patches, the former by setting the silver coverage to $0 \%$.

The CMY to CMYS conversion is performed in a maximum of three steps. First, we maximize the coverage of silver while keeping the $\Delta \mathrm{E}_{94}$ color difference between the original CMY color and the new CMYS color under the threshold $\Delta \mathrm{E}_{\max }$

$$
\begin{aligned}
& \left\{C_{1} M_{1} Y_{1} S_{1}\right\}=\underset{\{C M Y S\}}{\arg \max }[S] \text { such that } \\
& \qquad\left\{\begin{array}{l}
S \leq S_{\max } \\
\Delta \mathrm{E}_{94}\left[R_{\text {pred }}(\{C M Y S\}), R_{\text {pred }}\left(\left\{C_{0} M_{0} Y_{0}\right\}\right)\right]<\Delta \mathrm{E}_{\max } .
\end{array}\right.
\end{aligned}
$$

Then, if the resulting CMYS contains the maximum amount of allowed silver, i.e., $S_{\max }$, we minimize the coverage of yellow, while keeping silver to its maximum and while again keeping the $\Delta \mathrm{E}_{94}$ difference between the 


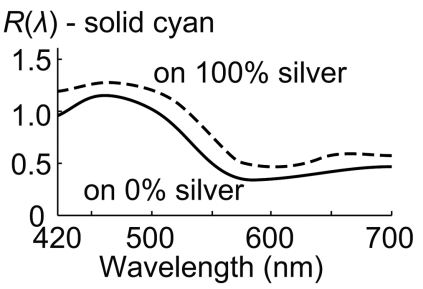

(a)

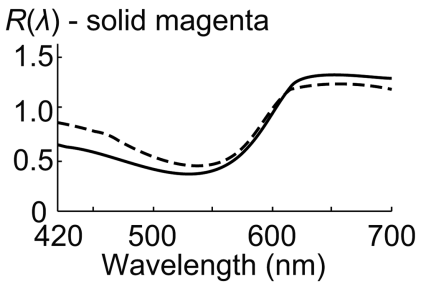

(b)

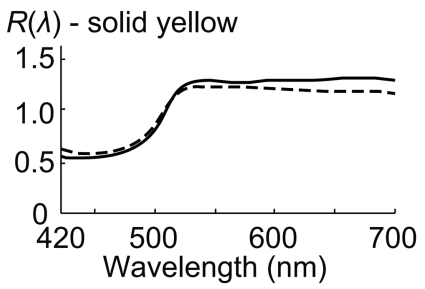

(c)

Figure 4. Measured specular reflectance factors $R(\lambda)\left(0^{\circ}: 0^{\circ}\right.$ geometry) of (a) solid cyan, (b) solid magenta, and (c) solid yellow when printed on paper (solid line) or on top of a solid layer of silver ink (dashed line).

original CMY color and the resulting CMYS color under the $\Delta \mathrm{E}_{\max }$ threshold

$$
\begin{aligned}
& \left\{C_{2} M_{2} Y_{2} S_{2}\right\}=\underset{\{C M Y S\}}{\arg \min }[Y] \text { such that } \\
& \qquad\left\{\begin{array}{l}
S=S_{1}=S_{\max } \\
\Delta \mathrm{E}_{94}\left[R_{\text {pred }}(\{C M Y S\}), R_{\text {pred }}\left(\left\{C_{0} M_{0} Y_{0}\right\}\right)\right]<\Delta \mathrm{E}_{\max } .
\end{array}\right.
\end{aligned}
$$

Finally, if the coverage $Y_{2}$ of yellow is reduced to $0 \%$, we minimize the $\Delta \mathrm{E}_{94}$ difference between the original CMY color and the resulting CMYS color while keeping silver to its maximum and yellow to $0 \%$

$$
\begin{gathered}
\left\{C_{3} M_{3} Y_{3} S_{3}\right\}=\underset{\{C M Y S\}}{\arg \min } \Delta \mathrm{E}_{94}\left[R_{\text {pred }}(\{C M Y S\}),\right. \\
\left.R_{\text {pred }}\left(\left\{C_{0} M_{0} Y_{0}\right\}\right)\right] \text { such that }\left\{\begin{array}{l}
S=S_{\max } \\
Y=0 \%
\end{array}\right.
\end{gathered}
$$

This algorithm is performed for all the 729 patches composed of all the combinations of cyan, magenta, and yellow at $0 \%, 13 \%, 25 \%, 37 \%, 50 \%, 63 \%, 75 \%, 87 \%$, and $100 \%$. These patches compose the CMY to CMYS conversion table; $S_{\max }$ is set to $100 \%$ and $\Delta \mathrm{E}_{\max }$ to 2 . Out of these 729 patches, 46 have reached the third step ( $\Delta \mathrm{E}_{94}$ minimization), 327 the second (yellow minimization), and 355 only the first (silver maximization). The remaining patch is the white patch that is not modified.

If we compare the resulting yellow coverages of the 373 patches reaching step 2 (yellow minimization) with the yellow coverages of the same patches when step 2 is skipped, we observe that the yellow coverages have been reduced by $9 \%$ on average ( $5 \%$ of the patches have a reduction of at least $18 \%$ and the maximum reduction is $25.6 \%$ ). If we exclude the 46 patches reaching step $3\left(\Delta \mathrm{E}_{94}\right.$ minimization), the yellow coverage is reduced by $9.7 \%$ on average. This is a significant reduction.

However, for 355 patches, the silver coverage could not be maximized. For 309 of these, one of the cyan, magenta, or yellow coverage is reduced to zero before silver reaches the upper bound. Since silver replaces similar amounts of cyan, magenta, and yellow, it is not possible to add more silver when there is no more of cyan, magenta, or yellow. The remaining 46 patches within this set of 355 patches are all originally composed of a solid layer of cyan. Since these colors are saturated, it is not possible to add much silver without inducing a large shift in chroma.

\section{EXPERIMENTAL RESULTS}

In order to verify the impact of reducing the amount of yellow, we choose 26 patches well distributed in the original CMY color space out of the 373 affected by the yellow reduction. We print three different versions of these patches: the original CMY patch, the corresponding CMYS patch when yellow reduction is applied, and the CMYS patch when yellow reduction is not applied. All the coverages are shown in Table III. In addition, the table shows two columns with measured $\Delta \mathrm{E}_{94}$ error between printed patches. The first column indicates the measured $\Delta \mathrm{E}_{94}$ error between the original CMY patches and their corresponding CMYS patches when yellow reduction is applied. The second column indicates the measured $\Delta \mathrm{E}_{94}$ error between the original CMY patches and their corresponding CMYS patches when yellow reduction is not applied.

The average measured $\Delta \mathrm{E}_{94}$ error for the first and second $\Delta \mathrm{E}_{94}$ columns are, respectively, 2.76 and 2.16. These averages are close to the expected $\Delta \mathrm{E}_{\max }=2$. The IS-YNSN model, therefore, performs very accurate predictions, especially considering that the conversion table is created in the

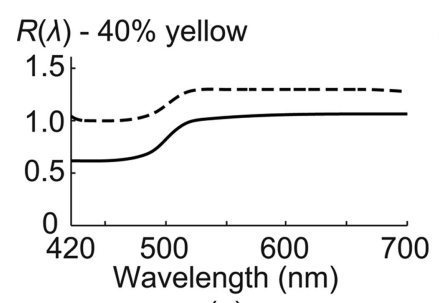

(a)

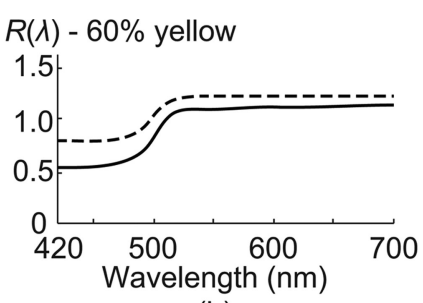

(b)

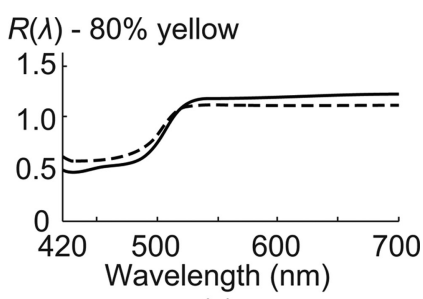

(c)

Figure 5. Measured specular reflectance factors $R(\lambda)\left(0^{\circ}: 0^{\circ}\right.$ geometry) of (a) $40 \%$ yellow, (b) $60 \%$ yellow, and (c) $80 \%$ yellow when printed on paper (solid line) or on top of a solid layer of silver ink (dashed line). 
Table III. Original CMY coverages of 26 patches and the corresponding CMYS coverages fitted by the IS-YNSN with or without yellow reduction. The coverages are given in percentages. The $\Delta \mathrm{E}_{94}$ error is measured between the printed original patch and the corresponding printed patch either when yellow reduction is applied or not.

\begin{tabular}{|c|c|c|c|c|c|c|c|c|c|c|c|c|c|}
\hline \multirow[b]{2}{*}{ Patch } & \multicolumn{3}{|c|}{ Original } & \multicolumn{5}{|c|}{ With yellow reduction } & \multicolumn{5}{|c|}{ Without yellow reduction } \\
\hline & C & M & Y & C & M & Y & s & $\Delta \mathrm{E}_{94}$ & C & M & Y & S & $\Delta \mathrm{E}_{94}$ \\
\hline 1 & 37 & 100 & 25 & 9.8 & 100.0 & 2.0 & 100.0 & 3.24 & 19.2 & 100.0 & 27.5 & 100.0 & 2.98 \\
\hline 2 & 75 & 100 & 25 & 65.5 & 100.0 & 3.1 & 100.0 & 2.26 & 71.0 & 100.0 & 25.1 & 100.0 & 2.53 \\
\hline 3 & 37 & 100 & 50 & 10.6 & 100.0 & 36.1 & 100.0 & 1.72 & 10.6 & 100.0 & 36.5 & 100.0 & 1.86 \\
\hline 4 & 87 & 75 & 25 & 86.7 & 69.8 & 3.9 & 100.0 & 2.43 & 87.8 & 74.5 & 24.7 & 100.0 & 2.21 \\
\hline 5 & 87 & 87 & 37 & 86.3 & 84.3 & 20.4 & 100.0 & 1.74 & 89.4 & 87.8 & 36.9 & 100.0 & 2.52 \\
\hline 6 & 37 & 75 & 25 & 9.0 & 71.4 & 2.8 & 100.0 & 3.56 & 17.3 & 70.6 & 21.2 & 100.0 & 1.31 \\
\hline 7 & 87 & 63 & 25 & 88.2 & 55.3 & 4.3 & 100.0 & 3.29 & 87.1 & 60.0 & 22.8 & 100.0 & 2.15 \\
\hline 0 & 25 & 37 & 75 & 0.0 & 26.7 & 63.9 & 100.0 & 2.53 & 0.0 & 29.4 & 76.1 & 100.0 & 1.55 \\
\hline 9 & 37 & 25 & 63 & 12.6 & 12.2 & 48.6 & 100.0 & 2.11 & 15.7 & 14.1 & 63.9 & 100.0 & 2.12 \\
\hline 10 & 25 & 100 & 63 & 0.0 & 96.5 & 53.7 & 100.0 & 0.82 & 0.0 & 98.4 & 59.6 & 100.0 & 1.44 \\
\hline 11 & 37 & 63 & 75 & 14.1 & 56.1 & 65.9 & 100.0 & 3.20 & 14.5 & 58.0 & 70.6 & 100.0 & 0.70 \\
\hline 12 & 25 & 25 & 75 & 0.0 & 11.4 & 63.9 & 100.0 & 2.21 & 0.0 & 14.1 & 76.1 & 100.0 & 1.50 \\
\hline 13 & 50 & 25 & 75 & 30.6 & 13.3 & 63.9 & 100.0 & 3.39 & 35.7 & 14.1 & 79.2 & 100.0 & 1.70 \\
\hline 14 & 25 & 50 & 63 & 0.0 & 40.8 & 51.0 & 100.0 & 2.86 & 0.0 & 45.5 & 64.7 & 100.0 & 2.87 \\
\hline 15 & 87 & 75 & 50 & 87.5 & 69.4 & 37.7 & 100.0 & 2.18 & 90.6 & 73.7 & 50.2 & 100.0 & 2.10 \\
\hline 16 & 87 & 63 & 50 & 88.6 & 54.9 & 38.0 & 100.0 & 3.33 & 90.6 & 60.8 & 49.8 & 100.0 & 2.29 \\
\hline 17 & 75 & 63 & 50 & 67.8 & 55.7 & 38.0 & 100.0 & 2.76 & 71.0 & 58.4 & 46.3 & 100.0 & 1.44 \\
\hline 18 & 63 & 25 & 75 & 49.4 & 13.3 & 65.1 & 100.0 & 3.17 & 55.3 & 13.7 & 77.7 & 100.0 & 1.81 \\
\hline 19 & 75 & 100 & 87 & 70.6 & 94.1 & 80.8 & 100.0 & 2.59 & 73.3 & 97.3 & 86.3 & 100.0 & 3.14 \\
\hline 20 & 87 & 37 & 25 & 90.6 & 24.3 & 5.1 & 100.0 & 4.03 & 86.3 & 29.8 & 17.7 & 100.0 & 3.30 \\
\hline 21 & 37 & 37 & 50 & 12.2 & 25.1 & 34.9 & 100.0 & 2.83 & 14.5 & 28.6 & 46.3 & 100.0 & 3.29 \\
\hline 22 & 75 & 50 & 25 & 68.2 & 40.0 & 4.3 & 100.0 & 3.51 & 68.2 & 43.5 & 18.0 & 100.0 & 2.55 \\
\hline 23 & 75 & 25 & 50 & 67.1 & 11.8 & 36.5 & 100.0 & 3.89 & 72.9 & 14.5 & 47.5 & 100.0 & 2.45 \\
\hline 24 & 63 & 75 & 75 & 51.4 & 69.0 & 67.8 & 100.0 & 2.53 & 54.9 & 73.3 & 76.5 & 100.0 & 1.20 \\
\hline 25 & 63 & 50 & 25 & 48.2 & 40.0 & 4.3 & 100.0 & 3.54 & 50.6 & 42.4 & 16.1 & 100.0 & 1.63 \\
\hline 26 & 25 & 50 & 37 & 0.0 & 39.2 & 21.2 & 100.0 & 2.13 & 0.0 & 42.8 & 30.6 & 100.0 & 3.43 \\
\hline
\end{tabular}

model calibration step based on measurements taken from a print session in February 2010, while the patches from Table III were printed at a different print sessions in December 2010. The lower average for the patches without yellow reduction is due to the fact that, when yellow reduction is skipped, we perform Eq. (8) instead. The $\Delta \mathrm{E}_{94}$ error between the prediction of the original CMY patch and the prediction of the fitted CMYS patch is then lower than the maximum $\Delta \mathrm{E}_{\max }=2$. This predicted $\Delta \mathrm{E}_{94}$ error for the 26 considered patches when yellow reduction is not applied is 1.40 on average. The $\Delta \mathrm{E}_{94}$ error measured on the corresponding printed patches is therefore on average larger by 0.76 both when yellow correction is applied $(2+0.76=2.76)$ and when yellow is not applied $(1.4+0.76=2.16)$.

Figure 6 shows the effect of reducing the yellow coverages on the specular reflectance factors of three of the 26 patches. For patch no. 5 (Fig. 6(a)), the reflectance factors of the original CMY patch and the CMYS patch fitted without reducing the yellow coverage are not distinguishable. However, when yellow reduction is applied, the difference in the blue region becomes noticeable. In this case, the yellow coverage has been reduced by $15 \%$.

For patch no. 9 (Fig. 6(b)), although the yellow coverage has been reduced by $15 \%$, there is not much difference 


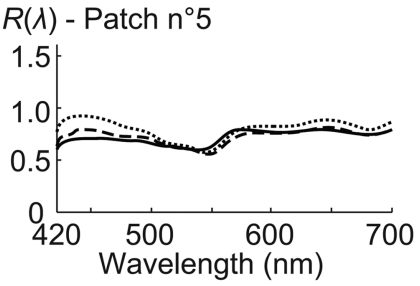

(a)

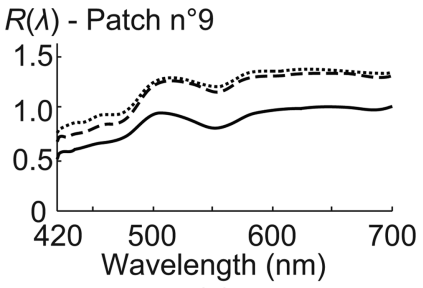

(b)

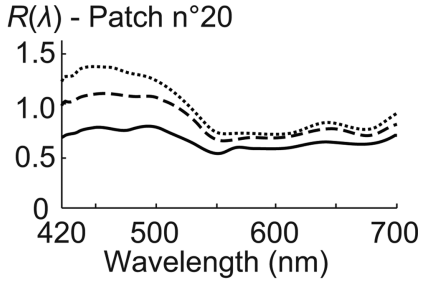

(c)

Figure 6. Measured specular reflectance factors $R(\lambda)\left(0^{\circ}: 0^{\circ}\right.$ geometry) of the original CMY patches (solid line), the fitted CMYS patches with yellow reduction applied (dotted line), and the fitted CMYS patches when yellow reduction is not applied (dashed line). The patches selected from Table III are (a) patch no. 5, (b) patch no. 9, and (c) patch no. 20.

between the two fitted CMYS patches and both can be distinguished from the original CMY patch. Finally, for patch no. 20 (Fig. 6(c)), the yellow coverage has been reduced by $12 \%$ and the impact of the yellow reduction is clearly visible in the blue region.

These patches are representative of the three different cases observed among the 26 selected patches. Yellow reduction can create a noticeable difference, increase an existing noticeable difference, or keep the existing noticeable difference. In no case has the yellow reduction decreased a noticeable difference or failed to create a noticeable difference. Pictures of the actual printed patches showing these different cases can be found in the Appendix.

Yellow reduction is, therefore, an important step when creating the MetallicArt conversion table. It ensures that the specular reflectance factors of the regions with and without silver can be differentiated. This is confirmed by the print trials we have performed. When yellow reduction was not applied, it was difficult to see the embedded patterns under specular viewing conditions. With the reduction applied, the patterns were clearly visible. However, since yellow reduction forces the $\Delta \mathrm{E}_{94}$ error close to the maximum error allowed, patterns located in uniform color areas may remain slightly visible under nonspecular viewing conditions.

\section{CONCLUSION}

MetallicArt is a technique to hide patterns in images using metallic ink under nonspecular viewing conditions and make them visible under specular viewing conditions. To keep the patterns hidden under nonspecular viewing conditions, the IS-YNSN spectral reflection prediction model is used. For the patterns to become visible under specular viewing conditions, a maximum of silver ink is included. This conversion strategy works well when the leafing effect can fully take place. This is, however, not the case on offset presses where drying occurs with UV curing inks. On such presses, the silver ink is UV cured as soon as deposited on paper. This reduces the leafing effect as well as the resulting specular reflection of the ink.

Since the reduced specular reflection of the silver ink cannot be differentiated from the specular reflection of the yellow ink, the embedded patterns are not visible anymore under specular viewing conditions in regions with large yellow surface coverages. We, therefore, propose a new CMY to CMYS conversion algorithm that not only maximizes silver but also minimizes yellow.

The experiments show that minimizing yellow increases the visibility of the patterns under specular viewing conditions. Moreover, thanks to the accuracy of the IS-YNSN model, the maximum $\Delta \mathrm{E}_{94}$ error tolerated is largely respected and the patterns remain hidden under nonspecular viewing conditions. Different print trials have demonstrated that this new MetallicArt technique works also on web offset presses when the leafing effect is reduced.

Although most of the print trials are successful, some designs work better than others. In uniform regions and smooth color gradients, it is more difficult to hide patterns. In these regions, the human eye perceives small color differences easily. A first improvement would be to apply MetallicArt with different parameters depending on the uniformity of the considered region, e.g., the $\Delta \mathrm{E}_{\max }$ parameter can be reduced to 1 in uniform or smoothly color-varying regions.

A second improvement would be to perform step 3 ( $\Delta \mathrm{E}_{94}$ minimization) for more patches. In the proposed algorithm, step 3 is performed only for patches whose yellow coverage has been reduced to $0 \%$ in step 2 (yellow minimization). However, as seen in Fig. 5(b), reducing yellow to $60 \%$ is sufficient. Step 3 can be applied to all the patches with a yellow coverage below $60 \%$ in order to improve the match between the original color and the fitted color under nonspecular viewing conditions. With this modification, 186 additional patches would have their $\Delta \mathrm{E}_{94}$ minimized.

It would be interesting to use more specular and nonspecular geometries, such as the ones recommended by the ASTM 2194 and DIN 6175-2 standards. It would be also interesting to verify the impact of various illuminants on the MetallicArt effect, e.g., by using the metameric index. ${ }^{10}$

\section{ACKNOWLEDGMENTS}

The authors thank the team of Perfect SA, located in Etoy, Switzerland, for allowing us to print cyan, magenta, yellow, and silver color images and patches on their web offset press. The authors also thank the Swiss National Science Foundation for their support under Grant No. 200020-126757/1.

\section{APPENDIX}

Figure A1 shows the pictures of 6 patches from the 26 selected and taken at near-specular viewing conditions. For 


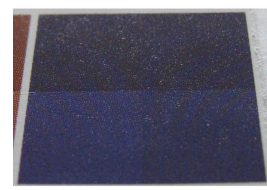

(a) Patch $n^{\circ} 5$

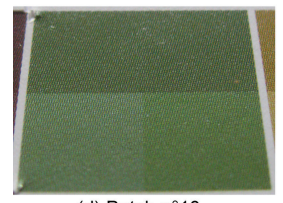

(d) Patch $n^{\circ} 18$

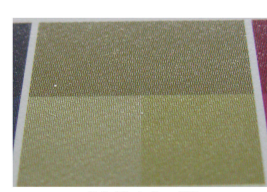

(b) Patch $n^{\circ} 9$

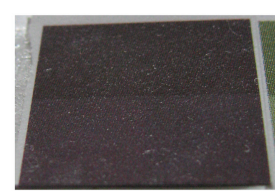

(e) Patch $n^{\circ} 19$

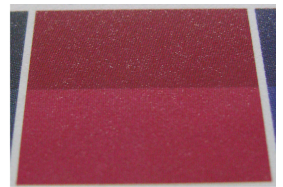

(c) Patch $n^{\circ} 10$

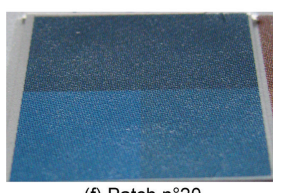

(f) Patch $n^{\circ} 20$
Figure A1. Pictures of (a) patch no. 5, (b) patch no. 9, (c) patch no. 10, (d) patch no. 18, (e) patch no. 19, and (f) patch no. 20 taken with a Canon PowerShot SX100 IS at near-specular viewing conditions. For each patch, the top, bottom-left, and bottom-right parts correspond, respectively, to the original CMY patch, the CMYS patch fitted with yellow reduction, and the CMYS patch fitted without yellow reduction.

patch no. 5(a), the difference between the original CMY patch at the top and the CMYS patch without yellow reduction at the bottom-right is barely noticeable. In comparison, the difference between the original CMY patch and the CMYS patch with yellow reduction at the bottom-left is more noticeable, as expected from the specular reflectance factors shown in Fig. 6(a). For patch no. 9(b), the difference between the original CMY patch and both CMYS patches is clearly visible and the slight color shift between both CMYS patches can also be seen in Fig. 6(b) around $450 \mathrm{~nm}$. Again, the picture of patch no. 20(f) shows the differences expected based on the specular reflectance factors shown in Fig. 6(c), i.e., the difference between the original CMY patch and CMYS patch with yellow reduction is larger than the already noticeable difference between the original CMY patch and the CMYS patch without yellow reduction.

Patches no. 10(c), no. 18(d), and no. 19(e) are further examples of the three different cases. For patch no. 10, the yellow reduction does not increase the existing difference between the original CMY and fitted CYMS patches. Conversely, for patch no. 18, the yellow reduction significantly increases the existing difference between the original CMY and fitted CYMS patches. Finally, patch no. 19 is the patch with the least visible differences among all 26 selected patches. There is almost no difference between the original CMY patch and the CMYS patch without yellow reduction, but yellow reduction creates a small difference that greatly helps distinguishing between the regions with and without silver.

\section{REFERENCES}

${ }^{1}$ R. D. Hersch, F. Collaud, and P. Emmel, "Reproducing color images with embedded metallic patterns," Proc. SIGGRAPH: ACM Trans. Graphics, 22, 427-436 (2003).

${ }^{2}$ G. M. Kern, J. Micale, S. Lavelle, and P. Valenzuela, "Hiding power of aluminium pigments in printed ink films," Am. Ink Maker 68, 60-68 (1991).

${ }^{3}$ F. R. Clapper and J. A. C. Yule, "The effect of multiple internal reflections on the densities of halftone prints on paper," J. Opt. Soc. Am. 43, 600-603 (1953).

${ }^{4}$ R. D. Hersch and F. Crété, "Improving the Yule-Nielsen modified spectral Neugebauer model by dot surface coverages depending on the ink superposition conditions," Proc. SPIE 5667, 434-445 (2005).

${ }^{5}$ H. E. J. Neugebauer, "Die theoretischen Grundlagen des Mehrfarbendrucks," Zeitschrift fuer wissenschaftliche Photographie 36, 36-73 (1937) [translated by D. Wyble and A. Kraushaar in "The theoretical basis of multicolor letterpress printing," Color Res. Appl. 30, 323-331 (2005)].

${ }^{6}$ J. A. C. Yule and W. J. Nielsen, "The penetration of light into paper and its effect on halftone reproductions," Proc. TAGA 3, 65-76 (1951).

${ }^{7}$ J. A. C. Yule and R. Colt, "Colorimetric investigation in multicolor printing," Proc. TAGA 3, 77-82 (1951).

${ }^{8}$ J. A. S. Viggiano, "Modeling the color of multi-colored halftones," Proc. TAGA 42, 44-62 (1990).

${ }^{9}$ D. R. Wyble and R. S. Berns, "A critical review of spectral models, applied to binary color printing," Color Res. Appl. 25, 4-19 (2000); M. E. Demichel, Procédé 26, 17-21 (1924).

${ }^{10}$ H. S. Fairman, "Metameric correction using parametric decomposition," Color Res. Appl. 12, 261-265 (1987). 